\title{
Growth Performance and Meat Quality of Broiler Chicks Fed Germinated and Fermented Soybeans
}

\author{
Dan-Won Lee, Jin-Ho Shin, Jung-Min Park, Jae-Chul Song ${ }^{1}$, Hyung-Joo Suh², Un-Jae Chang ${ }^{3}$, \\ Byoung-Ki An, Chang-Won Kang, and Jin-Man Kim* \\ Department of Food Science and Biotechnology of Animal Resources, Konkuk University, Seoul 143-701, Korea \\ ${ }^{1}$ Department of Food and Nutrition, Ulsan University, Ulsan 640-749, Korea \\ ${ }^{2}$ Department of Food and Nutrition, Korea University, Seoul 136-703, Korea \\ ${ }^{3}$ Department of Food and Nutrition, Dongduk Women's University, Seoul 136-714, Korea
}

\begin{abstract}
This study was conducted to investigate the dietary effects of germinated and fermented (with Monascus pupureus) soybean screenings (GFS) on growth performance and meat quality in broiler chicken. A total of 750 1-day-old Ross $\times$ Ross male broiler chicks were randomly allocated into five groups (five replications with 30 birds each) and fed experimental diets for 5 wks as follows: Group 1, negative-control (antibiotics-free diet); Group 2, positive-control (negative-control with 10 ppm of Avilamycin); Group 3, negative-control with 0.3\% GFS; Group 4, negative-control with 0.5\% GFS; Group 5, negative-control with $1 \%$ GFS. The final body weight of each group fed a diet containing $1 \%$ GFS was significantly higher than that of the negative-control group. The feed conversion ratios of all groups fed diets containing GFS and the positivecontrol group were significantly improved compared to the negative-control group during the whole period $(p<0.05)$. The relative weights of various organs along with the activities of serum glutamic oxaloacetic transaminase (GOT) and glutamic pyruvic transaminase (GPT) were not influenced by the dietary treatments. The levels of total serum cholesterol in groups fed diets containing $0.5 \%$ and $1 \%$ GFS were more significantly lowered compared to those of the control groups without GFS $(p<0.05)$. There were no significant differences in the cecal microflora profiles among the groups. Further, the dietary treatments did not influence the physico-chemical properties of the edible meat, including the shear force, $\mathrm{pH}$, meat color (CIE L*, $\mathrm{a}^{*}$ and $\mathrm{b}^{*}$ ), and content of malondialdehyde (MDA). Cooking loss of breast muscle in the groups fed GFS was significantly lowered compared to that of the negative control group $(p<0.05)$. These results indicate that dietary GFS could improve growth performance in broiler chicken and may affect meat quality in some instances.
\end{abstract}

Key words: germinated and fermented soybean, growth performance, blood cholesterol, broiler chicken

\section{Introduction}

Legumes are cultivated for their seeds, and are also called pulses. Their seeds are used for human and animal consumption or for the production of oils for industrial uses. Legumes, including beans, lentils, lupins, peas, and peanuts, have been cultivated for thousands of years, although many of the varieties of beans and peas that are commonplace today had been unknown until recently. Legumes play a major role in the traditional diets of many regions throughout the world. It is hard to think of the cuisines of Asia, India, South America, the Middle

*Corresponding author: Jin-Man Kim, Department of Food Science and Biotechnology of Animal Resources, Konkuk University, Seoul 143-701, Korea. Tel: 82-2-450-3688, Fax: 82-2-455-1044, E-mail: jinmkim@konkuk.ac.kr
East, and Mexico without picturing soybeans, lentils, black beans, chickpeas, and pinto beans, respectively. In contrast, in many Western countries, beans trend to play only a minor dietary role despite the fact that they have abundant nutrition values. So that, bean intake has decreased in the past century in most European countries (Hellendoorn, 1976).

Monascus purpureus (Van, 1884) is a homothallic fungus. It is classified under the Ascomycetes family Monascaceae. Among 24 known species of this genus, isolated from traditional oriental foods are characterized as from the genus Monascus, which is categorized into four species: M. pilosus, M. purpureus, M. rubber, and M. froridanus (Juzlova et al., 1996). Species of Monascus, which have been used in the production of traditional oriental foods similar to red mold rice, can produce different useful secondary metabolites, including red pigments, (natu- 
ral coloring agents), c-aminobutyric acid (GABA, antihypertensive), and monacolin $\mathrm{K}$ (MONK, a cholesterollowering drug) (Juzlova et al., 1996). Some Monascus compounds have applications as pharmaceuticals or food additives (Kraiak et al., 2000). In the former case, monacolin $\mathrm{K}$ was found to inhibit cholesterol synthesis and thus, to reduce hypolipidemia (Chairote et al., 2008; Lee et al., 2007). Previous studies showed that Monascus purpureus significantly reduced the low density lipoproteincholesterol (LDL-C), total cholesterol, triglycerides, and apolipoprotein B levels, and was well tolerated in patients with hyperlipidemia (Huang et al., 2007; Lin et al., 2005).

Seeking to improve the nutritive value of soybeans, preparation techniques have been developed to raise the bioavailability of their nutrients. (Chah et al., 1975) reported that diets containing soybeans fermented by Aspergillus oryzae significantly improved weight gain and feed efficiency in broiler chicks. Germination processes have been also used to increase the nutritive value and health qualities of the seeds (Urbano et al., 2005; Zanabria et al., 2006). Germination induces a substantial increase in the concentration of estrogenic compounds and all phytosterols, particularly â-sitosterol. But only limited information is available on the data about feeding of germinated and fermented soybeans in poultry.

In addition, the extensive uses of subtherapeutic antibiotics to improve growth and prevent infectious diseases have led to problems of drug residues in animal products and emerge of new antibiotic-resistance bacteria. In this regard, the use of antibiotics in animal feed was less common and endeavors are made to alternate means, in place of antibiotics, to prevent intestine from infectious diseases. The objectives of this experiment were to evaluate the growth performance, meat characteristics and physiological parameters in broiler chicks fed non-medicated diets containing germinated and fermented soybean screenings.

\section{Materials and Methods}

\section{Preparation of unmarketable soybeans}

Unmarketable soybean varieties commonly grown in Yonchon, Korea were obtained for use in this study. The soybeans were washed three times in distilled water at an ambient temperature for $10 \mathrm{~h}$. The soaked soybeans were drained and germinated in an incubator at $20^{\circ} \mathrm{C}$ for about $38 \mathrm{~h}$, and then cooked in an autoclave at $121^{\circ} \mathrm{C}$ for 20 min. The cooked soybeans were cooled to room temperature and inoculated with Monascus purpureus KCCM
12002, mixed, and fermented in an incubator for $7 \mathrm{~d}$. Then the fermented samples were dried in a hot-air oven at $60^{\circ} \mathrm{C}$ for $24 \mathrm{~h}$. After the drying, the germinated and fermented soybeans (GFS) were stored at $4^{\circ} \mathrm{C}$ until they were used.

\section{Experimental design and diets}

All the procedures were performed at the Department of Food Science and Biotechnology of Animal Resources of Konkuk University and met the requirements of the departmental standard. A total of 1-d-old 750 Ross male broiler chicks were used. The chicks were divided into five groups (five treatment groups $\times$ five replication groups $\times 30$ birds each) and fed the following diets for five wk. Group 1 was the negative control group (antibiotics-free); Group 2, the positive control group (negative control with $10 \mathrm{ppm}$ of Avilamycin); Group 3, the negative control group with $0.3 \%$ GFS; Group 4, the negative control group with $0.5 \%$ GFS; and Group 5, the negative control group with $1 \%$ GFS. The formula and chemical composition of the basal diets are shown in Table 1. GFS was substituted at the expense of control diet at $0.3,0.5$ or $1 \%$ levels on weight basis. Other nutrients and energy levels met or exceeded the minimum requirements of the National Research Council (NRC, 1994). The chicks were provided free access to the feeds and water. The feed intake and body weight of each group were recorded weekly. All animal care procedures were approved by the Institutional Animal Care and Use Committee at Konkuk University. At the end of the experiment period, eight chicks from each group were selected, weighed individually and then killed by asphyxiation with carbon dioxide. At the necropsy, the immune organs (the spleen and bursa of Febricius), abdominal fat, breast muscle, and leg muscle were immediately removed and weighed.

\section{Blood profiles}

At the end of the experiment period, eight chicks from each group were selected and weighed individually. About $8 \mathrm{~mL}$ of the blood was drawn from the jugular vein using a syringe to determine the concentration of various lipid fractions and components. The serum was separated from each blood sample via centrifugation and stored at $-30^{\circ} \mathrm{C}$ until it was used. The concentration of the total cholesterol (Total-C) and the activity of the glutamicoxaloacetic transaminase (GOT) and the glutamic-pyruvic transaminase (GPT) in the serum were measured according to the colorimetric method using cholesterol diagnostic kits (Cholesterol E kit, Youngdong Medical 
Table 1. Formula and chemical composition of the basal diets

\begin{tabular}{lcc}
\hline \multicolumn{1}{c}{ Ingredients } & Starter & Finisher \\
\hline Yellow corn & 47.35 & 48.84 \\
Wheat bran & 10.00 & 10.00 \\
Soybean meal & 21.76 & 19.97 \\
Corn gluten meal & 6.15 & 5.57 \\
Tallow & 3.75 & 4.85 \\
Rice polishing & 2.50 & 2.50 \\
Rapeseed meal & 5.00 & 5.00 \\
Vit.+Min. mixture ${ }^{1)}$ & 0.22 & 0.20 \\
L-lysine HCl (78\%) & 0.17 & 0.16 \\
DL-methionine (99\%) & 0.19 & 0.03 \\
Dicalcium phosphate & 1.53 & 1.28 \\
Limestone & 1.05 & 1.21 \\
Choline-Cl (50\%) & 0.07 & 0.10 \\
Salt & 0.25 & 0.25 \\
L-threonine (100\%) & 0.01 & 0.03 \\
\hline \multicolumn{1}{c}{ Total } & 100 & 100 \\
\hline Calculated values & & \\
TMEn ${ }^{2}$, kcal/kg & 3,160 & 3,220 \\
Crude protein, \% & 20.50 & 19.49 \\
Crude fat, \% & 6.04 & 6.90 \\
Ca, \% & 0.90 & 0.90 \\
Total P, \% & 0.66 & 0.62 \\
Available P, \% & 0.40 & 0.35 \\
Lysine, \% & 1.07 & 1.02 \\
\hline
\end{tabular}

${ }^{1)}$ The Vit.+Min. mixture provided the following nutrients per $\mathrm{kg}$ of diet: vitamin A, 18,000 IU; vitamin $\mathrm{D}_{3}, 3,750 \mathrm{IU}$; vitamin $\mathrm{E}$, $30 \mathrm{IU}$; vitamin $\mathrm{K}_{3}, 2.7 \mathrm{mg}$; vitamin $\mathrm{B}_{1}, 3.0 \mathrm{mg}$; vitamin $\mathrm{B}_{2}, 9.0$ mg; vitamin $B_{6}, 4.5 \mathrm{mg}$; vitamin $B_{12}, 30.0 \mu \mathrm{g}$; niacin, $37.5 \mathrm{mg}$; pantothenic acid, $15 \mathrm{mg}$; folic acid, $1.5 \mathrm{mg}$; biotin, $0.07 \mathrm{mg}$; $\mathrm{Fe}$, $75.0 \mathrm{mg}$; Zn, $97.5 \mathrm{mg}$; Mn, $97.5 \mathrm{mg}$; $\mathrm{Cu}, 7.5 \mathrm{mg}$; I, $1.5 \mathrm{mg}$; and Se, $0.2 \mathrm{mg}$.

${ }^{2)}$ TMEn, nitrogen corrected true metabolizable energy.

Corporation, Korea) and GOT-GPT assay kits (GOT-GPT assay kit, Youngdong Medical Corporation, Korea).

\section{Cecal microbial population}

The cecal digesta homogenates in the PBS were serially diluted from $10^{-1}$ to $10^{-7}$. The dilutions were subsequently plated on duplicate selective agar media for the enumeration of the target bacterial strains. The total microbes, coliforms, and Lactobacillus spp. were enumerated using nutrient agar, MacConkey agar, and MRS agar, respectively, via traditional methods (Tuohy et al., 2002). Each plate was incubated aerobically or anaerobically at $37^{\circ} \mathrm{C}$, for 24-72 h, after which the colonies were counted. The results were presented as base-10 logarithm colony-forming units per gram of cecal digesta.

\section{Meat quality}

The $\mathrm{pH}$ values of the breast muscle were measured in duplicate with a pH meter (340, Mettler-Toledo, Switzerland). About $5 \mathrm{~g}$ of the sample was cut into small pieces and homogenized with $20 \mathrm{~mL}$ of distilled water for $60 \mathrm{~s}$ in an Ultra-Turrax (T25, Janken \& Kunkel, Germany). The $\mathrm{pH}$ values were measured immediately after the homogenization. Objective color readings were determined in duplicate on a freshly cut breast muscle surface $24 \mathrm{~h}$ postmortem. The samples were placed on a styrofoam tray and over-wrapped with an oxygen-permeable film. After a $30 \mathrm{~min}$ bloom at $4^{\circ} \mathrm{C}$, the color was determined using a chromameter (CR 210, Minolta, Japan), which was set at CIE $L^{*}, a^{*}$ and $b^{*}$. The shear force values were determined with a Warner-Bratzler shear attachment on a texture analyzer (TA-XT2i, Stable Micro System Ltd., UK) with the following operating parameters: load cell, $50 \mathrm{~kg}$ and cross-head speed, $200 \mathrm{~mm} / \mathrm{min}$. Each core sample was sheared once across the center of the core, perpendicular to the muscle fiber. The shear force value was the mean of the maximum forces required to shear each set of core samples. Styrofoam, the $1.5 \mathrm{~cm}$ thick steaks that weighed about $80 \mathrm{~g}$ was put in a polyethylene bag. The packages were heated in a water bath at $75^{\circ} \mathrm{C}$ for $30 \mathrm{~min}$ and cooled at room temperature for $30 \mathrm{~min}$. The cooking loss percentage was determined from the steak weights before and after cooking.

The lipid oxidation was assessed on the basis of the malondialdehyde (MDA) that was formed during the refrigerated storage. The MDA, which is used as an index of lipid peroxidation, was determined using the selective third-order derivative spectrophotometric method (Botsoglou et al., 1994). Briefly, the meat samples were homogenized (Philips HP 2870, Philips, Eindhoven, Netherlands) in $8 \mathrm{~mL}$ of $5 \%$ aqueous trichloroacetic acid (Sigma Chemical Co., St. Louis, MO, USA) and $5 \mathrm{~mL}$ of $0.8 \%$ butylated hydroxytoluene in hexane, and the mixture was centrifuged. The top was discarded, and a 2.5 $\mathrm{mL}$ aliquot from the bottom layer was mixed with $2 \mathrm{~mL}$ of $0.8 \%$ aqueous 2-thiobarbituric acid, for further incubation at $70^{\circ} \mathrm{C}$ for $30 \mathrm{~min}$. After the incubation, the mixture was cooled under tap water and subjected to conventional spectrophotometry (DU 640 Beckman Coulter, Inc., USA) in the range of 400-650 nm. Third-order derivative spectra were produced via digital differentiation of the normal spectra using a derivative wavelength difference setting of $21 \mathrm{~nm}$. The concentration of the MDA in the analyzed samples $(\mu \mathrm{g} / \mathrm{g}$ meat) was calculated on the basis of the height of the third-order derivative peak at $521.5 \mathrm{~nm}$ by referring to the slope and intercept data of the computed least-squares fit of the standard calibration curve that was 
prepared using 1,1,3,3,-tetraethoxypropane, the precursor of MDA.

\section{Statistical analysis}

All the statistics were computed using the GLM procedures of the SAS software (SAS Institute, 2002). A software program that uses Duncan's multiple range test to compare treatment means was applied. One replicate was considered as the experimental unit for each performance parameter. The experimental unit was put on each bird for the other parameters. All the data were expressed as the mean \pm SD.

\section{Results and Discussion}

\section{Growth performance and carcass characteristics}

The dietary effects of the GFS on the growth of the broiler chicks are shown in Table 2. The final body weight of the positive-control group was the highest, but that of the chicks that were fed diets containing $1 \%$ GFS also significantly increased than in the negative-control group $(p<0.05)$. The dietary treatment did not have significant effects on the feed intake during growing and finishing stages. The daily BW gains of chicks significantly increased by supplementation of $0.3,0.5$ or $1 \%$ GFS as compared with negative-control group during finishing stage $(22-35 \mathrm{~d})(p<0.05)$. The positive-control and $1 \%$ GFS groups have shown higher the daily BW gain than that of the negative-control group during the whole period $(1-35 \mathrm{~d})(p<0.05)$. The feed conversion rate during the whole period in chicks fed diets containing GFS and positive-control group significantly improved than that of the negative-control group $(p<0.05)$.

The results of this study suggest that dietary GFS exerts an improvement in weight gain and feed efficiency and these are in agreement with those reported by Chah et al. (1975) with chickens and Zamora and Veum (1979) with growing pigs. Broiler chicks fed diets containing soybeans fermented by Aspergillus family had greater weight gain and feed efficiency as compared with those of untreated soybean group (Chah et al., 1975). Min et al. (2009) also reported that the apparent ileal $\mathrm{N}$ and amino acid digestibility were linearly increased as fermented soy protein levels were increased and a similar effect showed on growth performance in weaned pigs. It is assume that the growth promoting effect of GFS can be largely attributed to a greater supply of the essential amino acids and/ or a higher availability of nutrients. In this study, the GFS produced by $M$. purpureus family, at the level of $1 \%$, has the potential to improve the daily BW gain and feed conversion ratio and is a promising source for growth promotion in broiler diets.

The dietary treatment did not influence the relative weights of the liver, spleen, Bursa of Fabricius and breast and leg muscles (Table 3 ). The chicks fed diet containing $1 \%$ GFS had significantly lower $(p<0.05)$ relative abdominal fat weight, expressed as a percentage of the live BW, than those of negative- and positive-control groups. There is little research conducted with fermented soybean on body fat deposition. On the other hand, it has been known

Table 2. Dietary effects of germinated and fermented soybean (GFS) on the growth performance in broiler chicken ${ }^{1)}$

\begin{tabular}{|c|c|c|c|c|c|}
\hline & \multirow{2}{*}{ (-) Control } & \multirow{2}{*}{$(+)$ Control } & \multicolumn{3}{|c|}{ GGFS } \\
\hline & & & $0.3 \%$ & $0.5 \%$ & $1 \%$ \\
\hline Initial BW, g/bird & $40.4 \pm 1.24$ & $40.0 \pm 1.23$ & $40.4 \pm 1.28$ & $42.1 \pm 1.89$ & $40.6 \pm 1.16$ \\
\hline Final BW, g/bird & $1437.2 \pm 6.76^{\mathrm{b}}$ & $1526.7 \pm 18.86^{\mathrm{a}}$ & $1495.1 \pm 25.45^{\mathrm{ab}}$ & $1494.8 \pm 21.08^{\mathrm{ab}}$ & $1509.6 \pm 9.40^{\mathrm{a}}$ \\
\hline \multicolumn{6}{|c|}{ Feed intake, $\mathrm{g} / \mathrm{d} / \mathrm{bird}$} \\
\hline $1-21 d$ & $47.50 \pm 0.97$ & $47.36 \pm 0.93$ & $47.43 \pm 0.33$ & $47.82 \pm 0.84$ & $47.27 \pm 1.00$ \\
\hline $22-35 d$ & $136.83 \pm 0.82$ & $136.59 \pm 0.64$ & $136.25 \pm 0.38$ & $136.23 \pm 0.96$ & $136.16 \pm 0.99$ \\
\hline $1-35 d$ & $76.02 \pm 0.46$ & $76.06 \pm 0.56$ & $76.55 \pm 0.59$ & $75.54 \pm 0.82$ & $75.89 \pm 0.61$ \\
\hline \multicolumn{6}{|l|}{ BW gain, g/d/bird } \\
\hline $1-21 d$ & $29.68 \pm 0.36$ & $31.41 \pm 2.47$ & $30.60 \pm 3.22$ & $30.55 \pm 0.65$ & $30.63 \pm 0.28$ \\
\hline $22-35 d$ & $77.35 \pm 0.53^{\mathrm{b}}$ & $82.71 \pm 1.29^{\mathrm{a}}$ & $81.20 \pm 3.68^{\mathrm{a}}$ & $81.11 \pm 2.10^{\mathrm{a}}$ & $82.57 \pm 0.94^{\mathrm{a}}$ \\
\hline $1-35 d$ & $45.05 \pm 0.19^{\mathrm{b}}$ & $47.95 \pm 1.73^{\mathrm{a}}$ & $46.92 \pm 2.41^{\mathrm{ab}}$ & $46.86 \pm 0.66^{\mathrm{ab}}$ & $47.39 \pm 0.33^{\mathrm{a}}$ \\
\hline \multicolumn{6}{|l|}{ FCR, feed/gain } \\
\hline $1-21 d$ & $1.62 \pm 0.03^{\mathrm{ab}}$ & $1.52 \pm 0.04^{\mathrm{c}}$ & $1.63 \pm 0.07^{\mathrm{ab}}$ & $1.67 \pm 0.05^{\mathrm{a}}$ & $1.58 \pm 0.03^{b c}$ \\
\hline $22-35 d$ & $1.73 \pm 0.04^{\mathrm{a}}$ & $1.66 \pm 0.02^{\mathrm{bc}}$ & $1.68 \pm 0.07^{\mathrm{abc}}$ & $1.71 \pm 0.03^{\mathrm{ab}}$ & $1.65 \pm 0.02^{\mathrm{c}}$ \\
\hline $1-35 d$ & $1.72 \pm 0.01^{\mathrm{a}}$ & $1.61 \pm 0.04^{b}$ & $1.64 \pm 0.07^{\mathrm{b}}$ & $1.64 \pm 0.02^{b}$ & $1.62 \pm 0.02^{b}$ \\
\hline
\end{tabular}

${ }^{1)} \mathrm{GFS}$, germinated and fermented soybean; (-) Control, antibiotics-free diet without GFS; (+) Control, medicated diet without GFS.

${ }^{\mathrm{a}, \mathrm{b}, \mathrm{c}}$ Values with different superscripts within a row differ significantly $(p<0.05)$. 
Table 3. Dietary effects of germinated and fermented soybean (GFS) on the relative weights of various tissues in broiler chicken ${ }^{1)}$

\begin{tabular}{|c|c|c|c|c|c|}
\hline & \multirow{2}{*}{ (-) Control } & \multirow{2}{*}{$(+)$ Control } & \multicolumn{3}{|c|}{ GFS } \\
\hline & & & $0.3 \%$ & $0.5 \%$ & $1 \%$ \\
\hline & \multicolumn{5}{|c|}{ 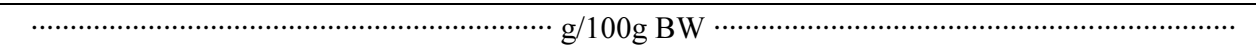 } \\
\hline Spleen & $0.10 \pm 0.01$ & $0.12 \pm 0.02$ & $0.11 \pm 0.01$ & $0.11 \pm 0.01$ & $0.12 \pm 0.01$ \\
\hline Liver & $2.22 \pm 0.09$ & $2.19 \pm 0.06$ & $2.43 \pm 0.09$ & $2.27 \pm 0.07$ & $2.33 \pm 0.06$ \\
\hline Bursa of Fabricius & $0.17 \pm 0.05$ & $0.18 \pm 0.03$ & $0.17 \pm 0.05$ & $0.19 \pm 0.07$ & $1.19 \pm 0.04$ \\
\hline Abdominal fat & $1.60 \pm 0.12^{\mathrm{a}}$ & $1.68 \pm 0.11^{\mathrm{a}}$ & $1.53 \pm 0.17^{\mathrm{ab}}$ & $1.57 \pm 0.15^{\mathrm{ab}}$ & $1.42 \pm 0.17^{\mathrm{b}}$ \\
\hline Breast muscle & $7.64 \pm 0.59$ & $7.90 \pm 0.21$ & $7.72 \pm 0.55$ & $7.71 \pm 0.47$ & $7.36 \pm 0.45$ \\
\hline Leg muscle & $9.06 \pm 0.96$ & $9.31 \pm 0.78$ & $9.23 \pm 0.43$ & $9.33 \pm 0.41$ & $9.23 \pm 0.47$ \\
\hline
\end{tabular}

${ }^{1)} \mathrm{GFS}$, germinated and fermented soybean; (-) Control, antibiotics-free diet without GFS; (+) Control, medicated diet without GFS.

${ }^{\mathrm{a}, \mathrm{b}}$ Values with different superscripts differ significantly $(p<0.05)$.

that dietary soy isoflavones is effective in lowering fat deposition in animals due to hormone-like action. Ali et al. (2004) have shown that soy isoflavones lowered fat deposition in four fat depots measured in experimental animals of obesity and diabetes. The abdominal fat pad weight was also decreased in the broiler chicken that was fed the low CP-amino acid diet with isoflavones (Payne et al., 2001b). It is obscure whether GFS isoflavones or combined with other protein fractions has effects on the reduction of abdominal fat, which need to be researched further.

\section{Cecal microbial population and blood profiles}

The profiles of the cecal microbes and blood metabolite are shown in Table 4. The number of total microbes, coliform bacteria, and lactic acid bacteria were not influenced by dietary treatments. The dietary treatment did not have significant effects on the activities of the GOT and GPT (Table 4). The serum enzymes measured are used as indicators of tissue damage because of exposure to certain toxins or anti-nutritional factors in avian species
(Lumeij, 1997). In addition, serum GOT and GPT is a valuable tool to determine a safe inclusion level for nonconventional feedstuff (Diaz et al., 2003). Thus, this result suggests that the GFS appeared safe at an inclusion rate of $1 \%$ without having adversary effects on physiological statue. The concentrations of serum total cholesterol in chicks fed diets containing 0.5 or $1 \%$ GFS were significantly lower than those of the negative- and positive-control groups $(p<0.05)$.

The reduction of the blood's total and LDL cholesterol concentrations with the consumption of products that contain soy protein has been shown repeatedly in humans and several animal models (Goodman-Gruen and KritzSliverstein, 2001). The exact component that is responsible for this action has yet to be identified. Isoflavone have been proposed to be the active ingredient that is responsible for the hypocholesterolemic effects of soy (Demonty et al., 2003). A previous study suggested that isoflavonerich soy protein is considerably more effective than isoflavone-depleted soy protein, though this finding is controversial. The cholesterol-lowering effect is one of

Table 4. Dietary effects of germinated and fermented soybean (GFS) on the profiles of cecal microflora and blood in broiler chicken $^{1)}$

\begin{tabular}{lccccc}
\hline \hline & $(-)$ Control & $(+)$ Control & & \multicolumn{2}{c}{ GFS } \\
\cline { 3 - 5 } & & & $0.3 \%$ & $0.5 \%$ & $1 \%$ \\
\hline Cecal microflora & & & & \\
Total microbes, Log cfu/g & $6.23 \pm 0.51$ & $6.17 \pm 0.47$ & $6.41 \pm 0.62$ & $6.29 \pm 0.61$ & $6.21 \pm 0.45$ \\
Coli forms, Log cfu/g & $5.95 \pm 0.57$ & $5.44 \pm 0.44$ & $5.30 \pm 0.41$ & $5.47 \pm 0.32$ & $5.37 \pm 0.44$ \\
Lactic acid bacteria, Log cfu/g & $6.17 \pm 0.34$ & $6.12 \pm 0.27$ & $6.13 \pm 0.39$ & $6.31 \pm 0.26$ & $6.44 \pm 0.35$ \\
Blood & & & & \\
GOT, U/L & $210.85 \pm 1.88$ & $209.25 \pm 1.66$ & $195.75 \pm 1.66$ & $212.5 \pm 1.85$ & $207.60 \pm 1.60$ \\
GPT, U/L & $14.41 \pm 0.91$ & $14.34 \pm 1.06$ & $17.36 \pm 0.91$ & $16.53 \pm 1.17$ & $17.46 \pm 0.95$ \\
Total-C, mg/dL & $136.10 \pm 6.86^{\mathrm{a}}$ & $128.13 \pm 3.05^{\text {ab }}$ & $122.79 \pm 3.85^{\text {bc }}$ & $117.93 \pm 2.47^{\mathrm{c}}$ & $115.43 \pm 3.56^{\mathrm{c}}$ \\
\hline
\end{tabular}

${ }^{1)} \mathrm{GFS}$, germinated and fermented soybean; (-) Control, antibiotics-free diet without GFS; (+) Control, medicated diet without GFS; GOT, glutamic-oxaloacetic transaminase; GPT, glutamic-pyruvic transaminase.

${ }^{\mathrm{a}, \mathrm{b}, \mathrm{c}}$ Values with different superscripts differ significantly $(p<0.05)$. 
several proposed mechanisms by which soy reduces the risk of heart disease (Gardner et al., 2001). In a study that evaluated the effects of isoflavone-rich and isoflavonedepleted soy protein on the plasma lipid concentration in postmenopausal, moderately hypercholesterolemic women, the isoflavone-rich soy protein lowered the total and LDL cholesterol more than did the isoflavone-depleted soy protein, though no significant differences were observed in the levels of HDL cholesterol or triacylglycerol. More expensive research is needed to determine the effect of GFS on cholesterol levels, often in edible meats, in broiler chicks under varying rearing conditions.

\section{Meat quality}

The results of meat quality of breast muscles are shown in Table 5. There were no significant differences in the $\mathrm{pH}$ and CIE color score among groups. The data clearly show that the difference in shear force of breast muscles was not significant among groups $(p>0.05)$. The cooking loss of all chicks fed diets containing GFS and positivecontrol group was significantly decreased than that of negative-control group $(p<0.05)$. The concentration of MDA was slightly, but not significantly, decreased in breast muscles of 1\% GFS supplemented group.

There has been very little research conducted on the effects of processed soybean on meat quality in broiler chickens. The dietary effects of soy isoflavones on meat quality were also somewhat variable. Payne et al. (2001a) demonstrated that the $a^{*}$ and $b^{*}$ CIE color scores in growing-finishing pigs decreased linearly as dietary isoflavones increased, but not significantly. The addition of daidzein in the maternal diet during late gestation did not also affect the meat color of the progeny (Rehfeldt et al., 2007). But, the feeding of isoflavones resulted in some desirable alterations, such as an improvement of $\mathrm{L}^{*}$ value in broiler chickens (Jiang et al., 2007). Our results show that dietary GFS does not significantly affect the $\mathrm{pH}$ and the CIE color score. In previous study, Shin et al. (2008) also reported that the yolk color was not affected by dietary GFS in laying hens.

The concentration of MDA in breast muscles was not affected by dietary treatment. The lack of effect of dietary GFS on muscle MDA level was somewhat unexpected, because isoflavone supplementation has been reported to frequently lower the MDA in the tissues and plasma (Yousef et al., 2004). Soybean isoflavone is effective in suppressing the formation of plasma lipid oxidation products in in vivo (Tikkanen et al., 1998; Wiseman et al., 2000). The concentrations of MDA in the plasma and tissues of male rabbits significantly decreased with isoflavone treatment (Yousef et al., 2004). Therefore, it is likely that the MDA-lowering effect of dietary soybean isoflavone does not always occur, because of differences in the isoflavone's optimum dose, feeding frequency, and duration of treatment. On the other hand, the cooking loss values in all the treated groups improved more significantly than that of negative-control group $(p<0.05)$. The cooking loss is influenced by various factors, including age, sex, diet, slaughter method, storage time and temperature, and meat properties (Lawrie, 1991). Oxidative processes may contribute to the loss of membrane integrity. It have been known that certain antioxidants such as selenium being beneficial to reduce drip loss by it's antioxidant property (Peric et al., 2009). Soy isoflavones have been shown to exert antioxidant activity (Ruiz-Larrea et al., 1997), and this could be suggested as a possible reason for the reduction in cooking loss by feeding of GFS. However, the levels of MDA in breast muscles have no significant change in our study, and thus further studies are required to clarify the role of GFS on oxidative pro-

Table 5. Dietary effects of germinated and fermented soybean (GFS) on the physic-chemical meat quality in broiler chicken ${ }^{1)}$

\begin{tabular}{|c|c|c|c|c|c|}
\hline & \multirow{2}{*}{ (-) Control } & \multirow{2}{*}{$(+)$ Control } & \multicolumn{3}{|c|}{ GFS } \\
\hline & & & $0.3 \%$ & $0.5 \%$ & $1 \%$ \\
\hline $\mathrm{pH}$ & $6.10 \pm 0.06$ & $6.17 \pm 0.07$ & $6.11 \pm 0.06$ & $5.97 \pm 0.09$ & $5.96 \pm 0.03$ \\
\hline \multicolumn{6}{|l|}{ CIE color score } \\
\hline $\mathrm{L}^{*}$ & $51.75 \pm 0.85$ & $50.22 \pm 0.68$ & $52.36 \pm 0.94$ & $50.20 \pm 0.31$ & $52.42 \pm 0.70$ \\
\hline$a^{*}$ & $2.61 \pm 0.42$ & $2.53 \pm 0.44$ & $2.46 \pm 0.29$ & $2.43 \pm 0.24$ & $2.47 \pm 0.32$ \\
\hline $\mathrm{b}^{*}$ & $10.55 \pm 0.83$ & $10.40 \pm 0.89$ & $11.59 \pm 0.78$ & $10.80 \pm 0.44$ & $12.03 \pm 0.96$ \\
\hline Cooking loss (\%) & $19.80 \pm 0.26^{\mathrm{a}}$ & $17.56 \pm 0.37^{b}$ & $17.80 \pm 0.34^{\mathrm{b}}$ & $17.50 \pm 0.50^{\mathrm{b}}$ & $17.48 \pm 0.30^{\mathrm{b}}$ \\
\hline Shear force $(\mathrm{kg})$ & $2.21 \pm 0.10$ & $2.26 \pm 0.13$ & $2.33 \pm 0.15$ & $2.02 \pm 0.08$ & $2.31 \pm 0.12$ \\
\hline $\mathrm{MDA}, \mu \mathrm{g} / \mathrm{g}$ & $1.62 \pm 0.03$ & $1.62 \pm 0.04$ & $1.63 \pm 0.07$ & $1.61 \pm 0.05$ & $1.58 \pm 0.04$ \\
\hline
\end{tabular}


cesses in chicken meats. In conclusion, chicks fed diets containing GFS had shown more improved growth performance and lowered total blood cholesterol and cooking loss in breast muscles. These results demonstrate that dietary GFS exerts growth-promoting and quality-enhancing effects in broiler chickens.

\section{Acknowledgments}

This study was supported by the Gyeonggi-do Agricultural Research \& Extension Services Center and Gyeonggi-do soybean project for area-based development in agriculture through industry-academy-research instituted collaboration.

\section{References}

1. Ali. A., Velasquez, M. T., Hansen, C. T., Mohamed, A. I., and Bhathena, S. J. (2004) Effects of soybean isoflavones, probiotics, and their interactions on lipid metabolism and endocrine system in an animal model of obesity and diabetes. J. Nutr. Biochem. 15, 583-590.

2. Botsoglou, N. A., Fletouris, D. J., Papageorgiou, G. E., Vassilopoulos, V. N., Mantis, A. J., and Trakatellis, A. G. (1994) Rapid, sensitive, and specific thiobarbituri acid method for measuring lipid peroxidation in animal tissue, food, and feedstuff samples. J. Agric. Food Chem. 42, 1931-1937.

3. Chah, C. C., Carlson, C. W., Semeniuk, G., Palmerand, I. S., and Hesseltine, C. W. (1975) Growth-promoting effects of fermented soybeans for broilers. Poult. Sci. 54, 600-609.

4. Chairote, E, Chairote, G., Niamsup, H., and Lumyong, S. (2008) The presence and the content of monacolins in red yeast rice prepared from Thai glutinous rice. World J. Microbiol. Biotechnol. 24, 3039-3047.

5. Demonty, I., Lamarche, B., and Jones, P. J. H. (2003) Role of isoflavones in the hypocholesterolemic effect of soy. Nutr. Rev. 61, 189-203.

6. Diaz, G. J., Roldan, L. P., and Cortes, A. (2003) Intoxication of Crotalaria pallida seeds to growing broiler chicks. Vet. Hum. Toxicol. 45, 187-189.

7. Gardner, C. D., Newell, K. A., Cherin, R., and Haskell, W. L. (2001) The effect of soy protein with or without isoflavones relative to milk protein on plasma lipids in hypercholesterolemic postmenopausal women. Am. J. Clin. Nutr. 73, 738745.

8. Goodman-Gruen, D. and Kritz-Silverstein, D. (2001) Usual dietary isoflavone intake is associated with cardiovascular disease risk factor in postmenopausal women. J. Nutr. 131, 1202-1206.

9. Huang, C. F., Li, T. C., Lin, C. C., Liu, C. S., Shih, S. C., and Lai, M. M. (2007) Efficacy of Monascus purpureus Went rice on lowering lipid ratios in hypercholesterolemic patients. Eur. J. Cardiovasc. Prev. Rehabil. 4, 438-240.

10. Hellendoorn, E. W. (1976) Beneficial physiologic action of beans. J. Am. Diet Assoc. 69, 248-253.

11. Jiang, Z. Y., Jiang, S. Q., Lin, Y. C., Xi, P. B., Yu, D. Q., and $\mathrm{Wu}, \mathrm{T} . \mathrm{X}$. (2007) Effects of soybean isoflavone on growth performance, meat quality, and antioxidation in male broilers. Poult. Sci. 86, 1356-1362.

12. Juzlova, P., Martinkova, L., and Kern, V. (1996) Secondary metabolites of the fungus Monascus. a review. J. Ind. Microbiol. Biotechnol. 16, 163-170.

13. Kraiak, S., Yamamura, K., Irie, R., Nakajima, M., Shimizu, H., and Chim-Anage, P. (2000) Maximizing yellow pigment production in fed-batch culture of Monascus sp. J. Biosci. Bioeng. 90, 363-367.

14. Lawrie, R. A. (1991) Meat Science (5 ${ }^{\text {th }}$ ed.) Pergamon Press, Oxford, UK.

15. Lee, C. L., Hung, H. K., Wang, J. J., and Pan, T. M. (2007) Improving the ratio of monacolin $\mathrm{K}$ to citrinin production of Monascus purpureus NTU568 under Dioscorea medium through the mediation of $\mathrm{pH}$ value and ethanol addition. $J$. Agric. Food Chem. 55, 6493-6502.

16. Lin, C. C., Lil, T. C., and Lai, M. M. (2005) Efficacy and safety of Monascus purpureus Went rice in subjects with hyperlipidemia. Eur. J. Endocrinol. 153, 679-86.

17. Lumeij, J. T. (1997) In: Clinical Biochemistry of Domestic Animals (ed. J. J. Kanebo, J. W. Harvey, and M. L. Bruss, 5th) Avian Clinic. Biochem. Academic Press 857-883.

18. Min, B. J., Cho, J. H., Chen, Y. J., Kim, H. J., Yoo, J. S., Wang, Q., Kim, I. H., Cho, W. T., and Lee, S. S. (2009) Effects of replacing soy protein concentrate with fermented soy protein in starter diet on growth performance and ileal amino acid digestibility in weaned pigs. Asian-Aust. J. Anim. Sci. 22, 99-106.

19. NRC (National Research Council) (1994) Nutrients Requirements of Poultry. $9^{\text {th }}$ rev. ed. National Academic Press, Washington, DC.

20. Payne, R. L., Bidner, T. D., Southern, L. L., and Geaghan, J. P. (2001a). Effects of dietary soy isoflavones on growth, carcass traits, and meat quality in growing-finishing pigs. $J$. Anim. Sci. 79, 1230-1239.

21. Payne, R. L., Bidner, T. D., Southern, L. L., and McMillin, K. W. (2001b) Dietary effects of soy isoflavones on growth and carcass traits of commercial broilers. Poult. Sci. 80, 1201-1207.

22. Peric, L., Milosevic, N., Zikic, D., Kanacki, Z., Dzinic, N., Nollet, L., and Spring, P. (2009) Effect of selenium sources on performance and meat characteristics of broiler chickens. J. Appl. Poult. Res. 18, 403-409.

23. Rehfeldt, C., Adamovic, I., and Kuhn, G. (2007) Effects of dietary daidzein supplementation of pregnant sows on carcass and meat quality and skeletal muscle cellularity of the progeny. Meat Sci. 75, 103-111.

24. Ruiz-Larrea, M. B., Mohan, A. R., Paganga, G., Miller, N. J., Bolwell, G. P., and Rice-Evans, C. A. (1997) Antioxidant activity of phytoestrogenic isoflavones. Free Radic. Res. 26, 63-70.

25. SAS. (2002) SAS User's guide. Statistics, ver. 8.e, Statistical Analysis System Institute. Inc., Cary, NC. USA. 
26. Shin, J. H., Park, J. M., Bak, D. J., Jeon, W. M., Song, J. C., Kim, S. K., An, B. K., Kang, C. W., Jung, W. S., and Kim, J. M. (2008) Effects of germinated and fermented unmarketable soybean on laying performance and egg quality in laying hens. Korean. J. Food Sci. Ani. Resour. 28, 667-674.

27. Tikkanen, M. J., Wahala, K., Ojala, S., Vihma, V., and Adlercreutz, H. (1998) Effect of soybean phytoestrogen intake on low density lipoprotein oxidation resistance. Proc. Natl. Acad. Sci. USA 95, 3106-3110.

28. Tuohy, K. M., Ziemer, C. J., Klinder, A., Knobel, Y., PoolZobel, B. L., and Gibson, G. R. (2002) A human volunteer study to determine the probiotic effects of lactulose powder on human colonic microbiota. Microb. Ecol. Health Dis. 14, 165-173.

29. Urbano, G., Aranda, P., Vilchez, A., Aranda, C., Cabrera, L., and Porres, J. S. M. (2005) Effects of germination on the composition and nutritive value of proteins in Pisum sativum, L. Food Chem. 93, 671-679.

30. Van, T. P. (1884) Monascus, genre nouveau de l'ordre des Ascomycetes. Bulletin de la Société de France 31, 26-31.
31. Wiseman, H., O’Reilly, J. D., Adlercreutz, H., Mallet, A. I., Bowey, E. A., Rowland, I. R., and Sanders, T. A. (2000) Isoflavone phytoestrogens consumed in soy decrease $\mathrm{F}(2)$-isoprostane concentrations and increase resistance of lowdensity lipoprotein to oxidation in humans. Am. J. Clin. Nutr. 72, 395-400.

32. Yousef, M. I., Kamel, K. I., Esmail, A. M., and Baghdadi, H. H. (2004) Antioxidant activities and lipid lowering effects of isoflavone in male rabbits. Food Chem. Toxicol. 42, 14971503.

33. Zamora, R. G. and Veum, T. L. (1979) Whole soybeans fermented with Aspergillus oryzae and Phizopus oligosporus for growing pigs. J. Anim. Sci. 48, 63-68.

34. Zanabria, E. R., Katarzyna, N., De Jong, L. E. Q., Birgit, H. B. E., and Robert, M. J. N. (2006) Effect of food processing of pearl millet (Pennisetum glaucum) IKMP-5 on the level of phenolic, phytate, iron and zinc. J. Sci. Food Agric. 86, 1391-1398.

(Received 2010.10.13/Revised 1st 2010.12.3, 2nd 2010.12.6/ Accepted 2010.12.6) 